\title{
A Qualitative Investigation of 'Context', 'Enterprise Mobile Services' and the Influence of Context on User Experiences and Acceptance of Enterprise Mobile Services
}

\author{
Karen Carey and Markus Helfert \\ Dublin City University (DCU), \\ Glasnevin, Dublin 9, Ireland \\ Karen.carey6@mail.dcu.ie, \\ Markus.helfert@computing.dcu.ie
}

\begin{abstract}
Within this paper an account of a qualitative investigation into Enterprise Mobile Services and their 'Context' is recorded. Employing Qualitative Content Analysis, two taxonomies are derived; An Enterprise Mobile Service Taxonomy and a 'Context' Taxonomy. The researcher also investigates current Context factors which have been proven to influence users' experiences and the acceptance of Enterprise Mobile Services. These are highlighted in the 'Context' taxonomy. The researcher intends to further investigate the influence of Context on users' acceptance of Enterprise Mobile Services subsequently it is necessary to select appropriate Context criteria for inclusion. This paper describes this selection process; a focus group with Industry experts was conducted following the KJ method. The Context criteria to be further investigated were selected. The criteria which describe enterprise mobile services were also selected. The results of the qualitative investigation reveal Context items which may potentially influence the acceptance of Enterprise Mobile Services. The HCI and IS domains could benefit from further investigations into the influence of these Context items on users experiences and acceptance to allow for an even deeper understanding of the influence of Context.
\end{abstract}

Keywords: Enterprise Mobile Services, Context, User Experience, User Perceptions, User Acceptance.

\section{Introduction}

User acceptance is defined as the demonstrable willingness within a user group to employ information technology for the tasks it is designed to support, [1]. This is usually measured based on user's perceptions of the IT. According to [2] user experience is defined as a person perceptions and responses that result for use and or anticipated use of a product system or service, consequently users experiences are important to consider when investigating user acceptance. Research concerning potential factors which would 
affect user experiences and consequently user acceptance of Enterprise Mobile Services is scarce, [3]. [3] argues that Context is an important factor to consider when measuring the acceptance of mobile services. Nevertheless, scholars have suggested that the concept of "Context" is complex and there is a tendency to overlook characteristics of the Context in which a product is being used [4].

This paper is part of a larger PhD study which aims to further investigate the influence of Context on the acceptance of Enterprise Mobile Services. Within this paper an account of a qualitative investigation into Enterprise Mobile Services and their 'Context' is recorded. Employing Qualitative Content Analysis, two taxonomies are derived; An Enterprise Mobile Service Taxonomy and a 'Context' Taxonomy. The researcher also investigates current Context factors which have been proven to influence users' experiences and the acceptance of Enterprise Mobile Services. These are highlighted in the Context taxonomy. As the researcher intends to further investigate the influence of Context on users experience and consequently the acceptance of Enterprise Mobile Service, it is necessary to select appropriate criteria for inclusion. To select these criteria a focus group with Industry experts was conducted following the $\mathrm{KJ}$ method, an account of this approach and the results are detailed within this paper.

This qualitative investigation emphasizes key Context items which require further investigation; this study will hopefully stimulate further research in these domains, the results of which will expand both the scholarly body of knowledge, but also have direct and tangible benefits for everyday users of Enterprise Mobile Services.

\section{Related Work}

The Technology Acceptance Model (TAM), [5-6] is one of the most widely accepted acceptance models in Information Systems literature. TAM has been tested in some domains of e-business and proved to be quite reliable to predict user acceptance of some new information technologies such as the intranet [7], World Wide Web [8] electronic commerce [9] and online shopping [10]. There are two primary factors in TAM: perceived usefulness (PU) and perceived ease of use (PEOU) that are of particular importance to determine user intention of adopting a new technology or information system. $\mathrm{PU}$ is defined as the degree to which a person believes that using a particular system would enhance his or her job performance [6]. PEOU is defined as the extent to which a person believes that using a particular system would be free from effort [6]. While acceptance of IT services has been one of the most prevailing IS research topics (e.g. $[6 ; 11 ; 12])$ the pervasiveness of mobile business raises new questions in exploring the adoption of Enterprise Mobile Services, such as what are the key factors determining the adoption of enterprise mobile services and how context factors affect user adoption of mobile services. [3], extended the TAM and constructed a mobile services acceptance model [13]. In addition to perceived ease of use and perceived usefulness, the mobile services acceptance model includes Trust, Context and Personal Initiatives and Characteristics Factors to study user acceptance of mobile services.

Within their model, Context is described as; any information that can be used to characterize the situation of entities (i.e. a person, place or object) that are considered relevant to the interaction between a user and an application, including the application and the user themselves, [14]. Based on their definition, Context can be viewed as a composite construct. 
Scholars have argued that the concept of "Context" is complex and there is a tendency to overlook characteristics of the Context in which a product is being used, [4]. Consequently there is a need to further investigate the Context of Enterprise Mobile Services and its influence on user acceptance. To describe how the Context of Enterprise Mobile Services is scoped within this research, the researcher qualitatively codes (using content analysis) the literature and selects criteria that comprise Context. Along with this the researcher qualitatively codes the literature to scope Enterprise Mobile Services within this research. Two taxonomies are developed; a description of this procedure is outlined in section 3. Although impossible to derive a complete list of factors that would represent the context with which enterprise mobile services are operated this research provides comprehensive Taxonomy. The researcher also reviews relevant literature to highlight Context items which have been included and measured in existing instruments which measure the influence of context on the acceptance of mobile services, these items are discussed further in section 4 .

\section{Investigating 'Context', 'Enterprise Mobile Services' and the Influence of Context on the Acceptance of Enterprise Mobile Services}

This paper qualitatively investigates 'Enterprise Mobile Services' and their 'Context'. The researcher also investigates current Context factors which have been proven to influence users' experiences and consequently the acceptance of Enterprise Mobile Services. As a result this paper addresses the following questions;

- What criteria scope the Enterprise Mobile Services?

- What criteria scope the Context of Enterprise Mobile Services?

- How is the influence of Context on the acceptance of mobile services currently measured?

- What additional Context measurement items are selected for further investigation?

To address the first three questions "Qualitative Content Analysis" is employed. Qualitative content analysis is one of the numerous research methods used to analyze text data. In this research, qualitative content analysis is defined as a research method for the subjective interpretation of the content of text data through systematic classification process of coding and identifying the themes or patterns, [15]. Two taxonomies are constructed; An Enterprise Mobile Service taxonomy comprising of the factors relating to Enterprise Mobile Services and A Context taxonomy; comprising of the factors relating to Context. Following this, a review of the literature was conducted to highlight those context factors which have been included in existing instruments which measure the acceptance of mobile services. To answer the final question a focus group with Industry Experts was conducted and the KJ method implemented to select the Context criteria that will be further investigated, [16]. The KJ method allows groups to quickly reach a consensus on priorities of subjective qualitative data. 


\subsection{Qualitative Content Analysis}

Initially the research objectives of the qualitative content analysis process were highlighted. Once these objectives were set, the researcher selected a sample of data sources to be analyzed. To avoid omission of key considerations, we conducted a comprehensive literature review and identified three domains (Information Systems, Human Computer Interaction, Human Factors and Ergonomics) that offer data sources that can contribute to the construction of the two taxonomies. By extensively analyzing a selection of significant contributions from the three overlapping domains, we mitigate the risk of overlooking key considerations. Providing a broad spectrum of sources enabled the researcher to construct the taxonomies which are reasonably comprehensive. Here we discuss the related research domains, explaining their relevance to the exercise of constructing the two taxonomies and identifying existing context items (that influence acceptance of mobile services) investigated.

\subsection{Data Sources}

Information Systems (IS) within the enterprise context capture and manage data to produce useful information that supports an organization and its employees, customer's suppliers, and partners, [17]. The term socio-technical systems was originally coined by [18] to describe systems that involve complex interaction between humans, machines and environmental aspects of the work system - nowadays this interaction is true of most enterprise systems. In recent years the term mobile service, mobilecommerce or enterprise mobile services have become a central topic in the Information Systems (IS) research community, [19]. Consequently, several contributions in the IS domain have committed to defining essential factors such as - people, machines and context - which need to be considered when developing such systems, ( [20]. Therefore sources within this domain are included in the data analysis. Additionally the IS field is committed to studding the factors which influence user intention to adopt new technologies. Over the years several models have been developed to test user attitude and intention to adopt new technologies. These models include; the Technology Acceptance Model [5-6]), Theory of Planned behavior (TPB) [21], Innovation Diffusion Theory, (IDT) [22] and the Unified Theory of Acceptance and Use of Technology (UTAUT), [23]. Subsequently sources within this domain are also included in the data analysis.

Human Computer Interaction (HCI) is concerned with the design, evaluation and implementation of interactive computing systems for human use and with the study of major phenomena surrounding them, [24]. Hence, HCI is concerned with enhancing the quality of interaction between humans and computer systems within the physical, organizational and social aspects of the users' environment to produce systems that are usable, safe and functional [25]. With the intention of providing further insight to design several contributions within this domain have also investigated people, machine and context characteristics which may restrict the interaction between humans and computer systems. Consequently sources within this domain are included in the data analysis.

Human Factors and Ergonomics is the multidisciplinary study of human biological, physical, psychological, and social characteristics in relation to environments, objects and 
services. The practice of human factors applies to the design, operation, and evaluation of "Systems" to ensure that that they are safe, efficient, comfortable and aesthetically pleasing to humans, [26]. Evidently overlap exists among the domains however, the main difference between Human Factors and Ergonomics and HCI is that HCI focuses more on users working specifically with computers, rather than other kinds of machines or design artifacts. There is also a focus in HCI on how to implement the computer software and hardware mechanisms to support human-computer interaction, thus Human Factors is a broader term. However contributions in this domain are relevant when describe the context of mobile services and also context factors which may influence these services. Therefore sources within this domain are included in the data analysis.

A keyword search was conducted using the following; Mobile Services, Enterprise Mobile Services, Context of Use, Perceptions of Mobile Services, Acceptance of Mobile Services, those articles deemed relevant by the researcher were included based on the following criteria;

- Must be related to mobile services.

- Must be related to Context of Mobile services.

- Must be related to users' perceptions of mobile services.

- Must be related to users' perceptions of mobile services in varying contexts.

- Must be related to users' acceptance/adoption of mobile services.

- Must be related to users' acceptance/adoption of mobile services in varying contexts.

From the three related domains, a total of (39) individual works are selected for inclusion in the analysis process. Out of the 39 individual works (13) will render the initial taxonomy for Enterprise Mobile Services and (26) individual works will render the initial taxonomy for Context. Whist examining the literature to identify contributions to highlight context characteristics that have been included in existing measurement instruments, the researcher could find only one data contribution which has focused on a thorough examination of an instrument (which includes context items) to measure the adoption of mobile services, and thus this source is included.

\subsection{Qualitative Coding Process}

Once the data sources were selected, the coding process commenced, the researcher followed the coding process described in [15], which involved four main steps; Open coding, Categorization of Codes, Coding on and Data reduction. An account of this process is recorded here.

\subsubsection{Open Coding}

Each line of the contributions was read by the researcher, and when a characteristic of an enterprise mobile service or the context of the service was apparent the researcher highlighted this part of the text and allocated a code. For example, Context has been previously defined as "The users, tasks, equipment (Hardware-software and materials) and the physical and social environments in which a product is used, [2]" consequently user's 'motion' was allocated a coded as it is a Context characteristic. During this stage each code was allocated a definition to ensure consistency of coding. This process continued until all studies were coded and a long list of initial codes existed. 


\subsubsection{Categorization of Codes}

This step involved renaming, merging, distilling and clustering related codes into broader categories of codes, consequently all codes related to mobile services were categorized under mobile services for example communication, Transaction etc. All codes related to Context were characterized under User, Task, Technology and Environment characteristics, for example for example, age, gender, skills were categorized under user. Also during this stage similar codes were clustered and renamed, for example codes such as display size and screen size were merged and renamed screen size. This continued until an initial taxonomy for both Enterprise Mobile Services and Context began to emerge and all related codes were structured under these taxonomies.

\subsubsection{Coding on}

This step involved breaking down the reorganized codes from step two into sub-codes so as to better understand the meanings embedded therein. For example under user categorization the user experience code was divided into the sub categories, novice intermittent and expert and under the task characteristics, task complexity was subdivided into low medium and high with a definition provided for each sub category. This step was continued until meanings were allocated to the each sub codes and the taxonomy began to represent taxonomy of Enterprise Mobile Services and taxonomy of Context.

\subsection{Data Reduction}

This step involved collapsing the coding tree/taxonomy into more abstract and generalized set of codes which represent enterprise mobile services and the Context of these services. This was done in conjunction with industry experts from an enterprise partner. The researcher arranged a focus group with industry experts, in doing so the KJ method was followed [16]. This includes six steps; an account of this process is summarized here.

\subsubsection{Determine a Focus Question}

It is necessary to outline the focus question as this drives the results. The researcher set the objective - to derive a list of criteria that would appropriately represent Enterprise mobile services and the Context of these services from an enterprise perspective. For a successful outcome it was important to the researcher that the participants would understand these objectives. The participants selected were industry experts in Small Form Factors and Human Factor Design. To ensure participants had a consistent understanding of the focus group objectives a presentation was given prior to the commencement of the focus group, 15 minutes covered the overall research aim and another 15 minutes for the aim of the focus group and the agenda.

\subsubsection{Organize the Group}

To organize the group members the two main sources in the industry partner were contacted and the intentions of the focus group were outlined. The researcher suggested that a diverse group of members would be beneficial to get different perspectives. A total of 5 participants were organized, these included small form factor and human factor specialists, a business analyst and a project manager, all who were familiar with enterprise mobile services. The time required was outlined and a date was set for 3 hours over 3 days (every Tuesday for 3weeks). 


\subsubsection{Put Data Onto Sticky Notes and Put Sticky Notes on the Wall}

After the presentation and the objectives of the focus group was outlined to members, the taxonomies of derived criteria were put onto yellow sticky notes and put on the walls in a large room. At the very top of the wall the focus group objectives were posted on orange paper, this was to ensure that members could keep focus. Participants were given pink sticky notes and told to read all sticky notes on the wall, if at any time they felt that something else should be added, something should be renamed or something should be removed they were asked to write this down on the pink sticky notes beside the criteria they felts should be revised.

\subsubsection{Group Similar Items and Name Each Group}

Once all opinions and contributions had been posted on pink sticky notes, participants were asked to group the criteria which they felt belonged in the same category. This involved moving all the criteria under the following five main criteria, Mobile Service, Context (Task, Technology, User, and Environment). Each of these main groups had a number of sub criteria, the naming and grouping of these sub-criteria were very important to the researcher as these would detail the mobile service and the context. The participants were asked to then read each group and review everything on the wall and consider its position. The results of this are illustrated in Appendix A and B. During this stage the literature was reviewed to highlight those context items that have been included in existing measurement instruments, these items are the shaded in criteria in Appendix B. It is evident in Appendix B that, user experience, attitude, safety, enjoyment, accessibility, compatibility, social factors and location have been included in existing measurement instruments, which measure the acceptance of mobile services.

The Context criteria in the Context taxonomy which are not highlighted include items which need further investigation. Consequently, this qualitative investigation emphasizes key Context items which require further examination; hopefully this will stimulate further research in these domains. As the researcher intends to further investigate a selection of these Context criteria, the selection process is detailed the next section. The researcher was also interested in scoping Enterprise Mobile Services from an Enterprise Perspective. This is also discussed in the next section.

\subsubsection{Voting for the Most Important Group}

After the participants had reviewed the revised and categorized criteria they were asked select the three most important criteria to them that represent Enterprise Mobile Services and then for Context. Each of the participants votes were recorded by the researcher.

\subsubsection{Ranking the Most Important Group}

Once all participants' votes were recorded, the researcher ordered each criterion selected by the number of votes they received, with the highest at the top. The participants were asked to review the selected criteria and discuss. At this stage conflicting selection was discussed and participants continued to move the sub categories around. After discussing and moving the sub-categories around to reach a unanimous agreement a final count of the votes allocated to each subcategories under the five main categories were counted, i.e. there are sub- criteria under each of the five main categories that are ranked much higher than the rest. At this point the process stopped as any further combinations are unlikely to change the top priorities voted by the participants. The focus group was 
declared finished and the researcher reviewed the final selection of criteria along with the continuous opinionated data recorded throughout the focus group process. The results of the final list of criteria to classify Enterprise Mobile Services and the Context criteria for inclusion are recorded in table 1.

Table 1. Selected Criteria from Focus group

\begin{tabular}{|l|c|l|c|l|l|l|l|l|l|}
\hline \multicolumn{2}{|c|}{$\begin{array}{c}\text { Enterprise Mobile } \\
\text { Services }\end{array}$} & \multicolumn{9}{|c|}{ Context Criteria } \\
\cline { 2 - 11 } & User & \multicolumn{2}{|c|}{ Task } & \multicolumn{2}{c|}{ Technology } & \multicolumn{2}{|c|}{ Environment } \\
\hline Criteria & $\%$ & Criteria & $\%$ & Criteria & $\%$ & Criteria & $\%$ & Criteria & $\%$ \\
\hline $\begin{array}{l}\text { Communica- } \\
\text { tion }\end{array}$ & 48 & $\begin{array}{c}\text { Cognitive } \\
\text { load }\end{array}$ & 52 & $\begin{array}{c}\text { Criticali- } \\
\text { ty }\end{array}$ & 8 & $\begin{array}{c}\text { Portabil- } \\
\text { ity }\end{array}$ & 8 & $\begin{array}{c}\text { Ambient } \\
\text { Condi- } \\
\text { tions }\end{array}$ & 32 \\
\hline Transaction & 25 & Motion & 20 & & & & & & \\
\hline Information & 24 & & & & & & & & \\
\hline Web 2.0 & 20 & & & & & & & & \\
\hline Learning & 16 & & & & & & & & \\
\hline
\end{tabular}

It is evident in table 1 that to scope Enterprise Mobile Services the criteria, Communication (48\%), Transaction (24\%), Information (24\%), Web $2.0(20 \%)$ and Learning (16\%) have been selected. Consequently Enterprise Mobile Services can be categorized under these. It also evident from table 1 that the selected criteria that describe the Context of these services and will be further investigated by the researcher include, User Cognitive load (52\%), Environmental Ambient conditions (32\%), User Motion (20\%), Task criticality (8\%) and Portability (8\%). Future work involves testing for convergent and discriminant validity of these criteria, testing the reliability of these criteria and to demonstrate the influence of these criteria on the acceptance of Enterprise Mobile Service Acceptance.

\section{Conclusions, Limitations and Future Work}

Within this paper an account of a qualitative investigation into Enterprise Mobile Services and their 'Context' is recorded. Employing Qualitative Content Analysis, two taxonomies are derived; An Enterprise Mobile Service Taxonomy and a Context Taxonomy. The researcher also investigates current Context factors which have been proven to influence users' experiences and the acceptance of Enterprise Mobile Services. These are highlighted in the Context taxonomy. As the researcher intends to further investigate (as part of a larger $\mathrm{PhD}$ project) the influence of Context on users experience and consequently the acceptance of Enterprise Mobile Service, it is necessary to select appropriate criteria for inclusion. To select these criteria a focus group with Industry experts was conducted following the KJ method, an account of the selection approach and the results are detailed within this paper. Future work involves testing the validity and reliability of these constructs while also investigating the influence of these Context criteria on the acceptance of Enterprise Mobile Service. Currently the researcher is planning a field study within an Enterprise partner. In conclusion this qualitative investigation has emphasized key Context items which 
require further investigation; these will hopefully stimulate further research in the IS and HCI domains, the results of which will expand both the scholarly body of knowledge, but also have direct and tangible benefits for everyday users of Enterprise Mobile Services.

\section{References}

1. Dillon, A., Morris, M.G.: User acceptance of information technology: theories and models. Journal of the American Society for Information Science 31, 3-32 (1996)

2. ISO 9241-210. Ergonomics of human system interaction Part 2 Human centred design for interactive systems (2010)

3. Gao, S., Krogstie, J., Siau, K.: Developing an Instrument to measure the adoption of Mobile Services. Mobile Information Systems 7, 1 (2011)

4. Alonso-Rios, D.: Usability a critical analysis and taxonomy. International Journal of Human-computer Interaction 26(1), 53-74 (2009)

5. Davis, F.: Perceived usefulness, perceived ease of use, and user acceptance of information technology. MIS Quarterly, 139-140 (1989)

6. Davis, F.D., Bagozzi, R.P., Warshaw, P.R.: User acceptance of computer technology: a comparison of two theoretical models. Management Science 35, 982-1003 (1989)

7. Horton, R.P.: Explaining Internet use with the technology accepatcne model. Journal of Information Technology 16, 281-298 (2001)

8. Lederer, A.L.: The technology acceptance model and the World Wide Web. Decision Support Systems 29, 269-282 (2000)

9. Pavlou, P.A.: Consumer Acceptance of Electronic Commerce: Integrating Trust and Risk with the Technology Acceptance Model. International Journal of Electronic Commerce 7, 101-134 (2003)

10. Gefen, D.: TAM or Just Plain Habit: A Look at Experienced Online Shoppers. Journal of End User Computing 15, 1-13 (2003)

11. Taylor, S., Todd, P.: Understanding Information Technology Ugase: A Test of Competing Models. Information Systems Research 6, 144-176 (1995)

12. Ven, K., Verelst, J.: The Impact of Ideology on the Organizational Adoption of Open Source Software. Journal of Database Managemnet 19, 58-72 (2008)

13. Gao, S., Krogstie, J., Gransaether, P.A.: Mobile Service Acceptance Model. In: Proceedings of the 2008 International Conference on Convergence and Hybrid Information Technology. IEEE Computer Society (2008)

14. Dey, A.K.: Understanding and Using Context. Personal Ubiquitous Computing 5, 4-7 (2001)

15. Krippendorff, K.: Content Analysis: An Introduction to its Methodology. Sage, Thousand Oaks (2004)

16. Spool, J.: The KJ-Technique: A Group Process for Esablishing Priorities, http://www.uie.com/articles/kj_technique/

17. Whitten, J.L., Barlow, V.M., Bentley, L.: Systems analysis and design methods. McGrawHill Professional (1997)

18. Emery, F.E., Trist, E.: LSocio-Technical systems. In: Chruchman, C.W. (ed.) Management Science Models and Techniques, Oxford, UK, vol. 2, pp. 83-97 (1960)

19. Wang, F.Y.: Social computing: from social informatics to social intelligence. IEEE Intelligent Systems 22(2), 79-83 (2007) 
20. Baxter, G., Sommerville, I.: Socio-tecnical system: From design methods to system engineering. Interacting with Computers 23(1), 4-17 (2011)

21. Ajzen, I.: The thepry of planed behavior. Organisational Behaviour and Human Decision Processes 50, 179-211 (1991)

22. Rogers, E.M.: The Diffusion of Innovations. Free Press, New York (1995)

23. Venkatesh, V.: User Acceptance of Information Technology: Toward a Unified View. MIS Quarterly, 425-478 (2003)

24. Zhang, P., Li, N.: An assessment of human-computer interaction research in management infomation systems: topics and methods. Computers in Human Behavior 20(2) (2004)

25. Preece, J.: Human Computer Interaction. Addison-Wesley, Harlow (1994)

26. Usability Body of Knowledge. Usability Body of Knowledge, http: / / www . usabilitybok.org/glossary

\section{Appendix A}

\begin{tabular}{|c|c|c|c|c|c|c|c|c|c|c|c|c|}
\hline & & \multicolumn{11}{|c|}{ Data Sources } \\
\hline Classification & Factors & $\mid$\begin{tabular}{l}
3 \\
$\vdots$ \\
0 \\
\hdashline
\end{tabular} & \multicolumn{2}{|c|}{ 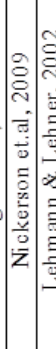 } & 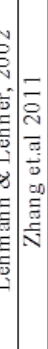 & \multicolumn{2}{|c|}{ 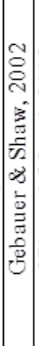 } & 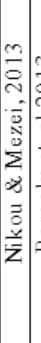 & & 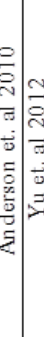 & $=$ & 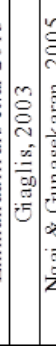 \\
\hline \multirow{22}{*}{ Mobile Services } & Communication & & & 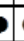 & & & 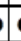 & & & $\bullet$ & $\bullet$ & \\
\hline & Entertainment & & $\bullet$ & & & & & & & & $\bullet$ & 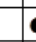 \\
\hline & Information & & & t & 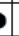 & 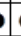 & & & & $\bullet$ & & 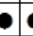 \\
\hline & Web 2.0 & & & & & & & & & $\bullet$ & & \\
\hline & Transaction & & & (5) & & & & & & $\bullet$ & - & 0 \\
\hline & Innovativeness & $\bullet$ & $\bullet$ & & & & & 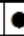 & & & & \\
\hline & Us age Context & $\bullet$ & & & & & & & & & & \\
\hline & Temporal & & & & & & & & & & & \\
\hline & Location Based & & & 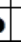 & & & & & $\bullet$ & $\bullet$ & & 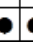 \\
\hline & Identity & & & 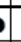 & & & & & & & & - \\
\hline & Multiplicity & & & & & & & & & & & \\
\hline & Public & & & & & & & & & & & \\
\hline & Interaction & & & & 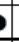 & & & & & & $\bullet$ & \\
\hline & Data Proces sing & & & & & & & & & & & \\
\hline & Credibility & & & & & & & 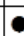 & & & & \\
\hline & Adaptivity & & & & & & & 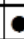 & & & & \\
\hline & Cost & & & & & & & 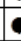 & & & & \\
\hline & Flexibility & & & & & & & 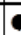 & & & $\bullet$ & \\
\hline & Personalisation & & & & & & & 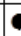 & & & & \\
\hline & Mobility & & & & & & & & $\bullet$ & & & \\
\hline & Learning & & & & & & & & & $\bullet$ & - & \\
\hline & Mess aging & & & & & & t & & & & $\bullet$ & \\
\hline
\end{tabular}




\section{Appendix B}

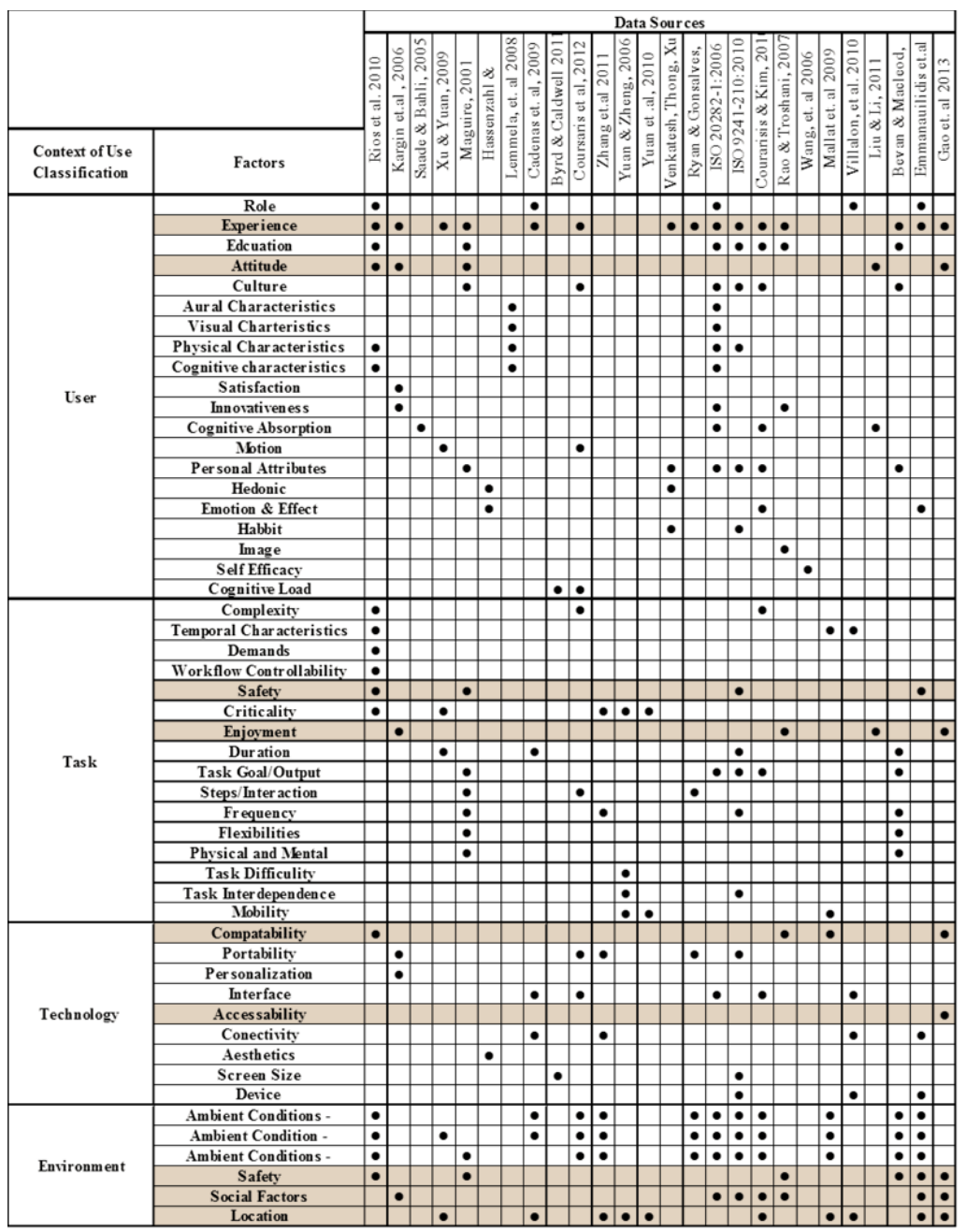

